\title{
Weekly vs. 3-weekly paclitaxel, carboplatin, and cetuximab (PCC) in recurrent/metastatic head and neck cancer
}

\author{
LEEANN GERAGHTY ${ }^{1}$, THOMAS E. SCHULTZ ${ }^{2}$, SARAH E. HOFFMAN ${ }^{2}$, KYLE PORTER $^{3}$, \\ MAJD ISSA $^{4}$, VIDHYA KARIVEDU ${ }^{4 *}$ and MARCELO BONOMI ${ }^{*}$ \\ ${ }^{1}$ Department of Pharmacy, Norton Cancer Institute, Louisville, KY 40241; ${ }^{2}$ Department of Pharmacy, \\ The James Cancer Hospital at The Ohio State University; ${ }^{3}$ Center for Biostatistics, Department of Biomedical Informatics, \\ The Ohio State University; ${ }^{4}$ Department of Internal Medicine, Division of Medical Oncology, \\ The James Cancer Hospital at The Ohio State University, Columbus, OH 43210, USA
}

Received April 30, 2021; Accepted August 6, 2021

DOI: $10.3892 / \mathrm{mco} .2021 .2403$

\begin{abstract}
The combination of paclitaxel, carboplatin and cetuximab (PCC) is efficacious in patients with recurrent/metastatic $(\mathrm{R} / \mathrm{M})$ squamous cell carcinoma of the head and neck (SCCHN). The current study assessed the incidence of grade 3/4 (G3/4) toxicity for patients receiving weekly or 3-weekly PCC for R/M SCCHN. The present single-institution, retrospective analysis included 74 patients who received weekly [paclitaxel $45 \mathrm{mg} / \mathrm{m}^{2}$ and carboplatin area under the curve (AUC), 1.5] or 3-weekly (paclitaxel $175 \mathrm{mg} / \mathrm{m}^{2}$ and carboplatin AUC, 5) PCC. For each regimen, cetuximab was administered at $400 \mathrm{mg} / \mathrm{m}^{2}$ for the first week, after which the dosage was reduced to $250 \mathrm{mg} / \mathrm{m}^{2}$ weekly until disease progression occurred. Toxicity was assessed according to the Common Terminology Criteria for Adverse Events v4.03, and response to therapy was determined using computed tomography every 12 weeks. The results revealed that 26 patients (35\%) received weekly PCC and 48 patients $(65 \%)$ received PCC every 3 weeks. A total of $6(25 \%)$ patients receiving weekly PCC experienced G3/4 toxicity compared with $30(66 \%)$ patients that received PCC every 3 weeks (odds ratio, $0.18 ; 95 \%$ confidence interval, $0.05-0.64 ; \mathrm{P}=0.01$ ). The most common G3/4 side effects were neutropenia (8 vs. $53 \%$ ), anemia (15 vs. $32 \%$ ) and fatigue (3 vs. $10 \%)$. The incidence of G3/4 toxicity or any grade toxicity requiring dose modification or discontinuation was 74 vs. $77 \%$, respectively. The overall response rate was $39 \%$ with weekly PCC compared
\end{abstract}

Correspondence to: Dr Marcelo Bonomi, Department of Internal Medicine, Division of Medical Oncology, The James Cancer Hospital at The Ohio State University, 1336 Lincoln Tower, 1800 Cannon Drive, Columbus, OH 43210, USA

E-mail: Marcelo.Bonomi@osumc.edu

*Contributed equally

Key words: chemotherapy, head and neck cancer, carboplatin, paclitaxel, cetuximab, treatment with $27 \%$ in those receiving PCC every 3 weeks. The 1 -year progression-free and overall survival rates were 27 and $46 \%$ for patients receiving weekly PCC, and 13 and $44 \%$ for patients receiving PCC every 3 weeks. Weekly PCC had a reduced risk of G3/4 toxicity when compared with PCC administered every 3 weeks. Considering the improved tolerance of weekly PCC, this regimen should be considered for older patients and patients being treated with second-line chemotherapy.

\section{Introduction}

In 2018, cancers of the head and neck accounted for approximately $3.7 \%$ of new cancer diagnoses in the United States, with about 64,700 new cases and 13,700 deaths (1). These cancers may occur at any site in the head and neck, with some of the more common sites including the oral cavity, pharynx, and larynx. Tobacco and alcohol abuse have historically been the most common risk factors associated with head and neck cancers, however cases associated with human papillomavirus (HPV) infection have been increasing (2). Up to $90 \%$ of head and neck cancers are of squamous cell carcinoma (SCC) histology, and over $90 \%$ of those have overexpression of epidermal growth factor receptor (EGFR) (1). While the majority of patients present with early stage or locally advanced disease, up to $15 \%$ of patients present with distant metastases (3). Prognosis in the recurrent or metastatic $(\mathrm{R} / \mathrm{M})$ setting is poor, with a median overall survival (OS) of less than one year (4).

Until recently, chemotherapy was used in the first-line setting for $\mathrm{R} / \mathrm{M}$ disease. The preferred regimen is based on results of the EXTREME trial $(1,5)$. In this study, 442 patients with R/M SCCHN were randomized to a standard chemotherapy regimen containing a platinum (cisplatin or carboplatin) plus 5-fluorouracil (5-FU), with or without cetuximab, an anti-EGFR monoclonal antibody. Patients in the cetuximab-containing arm had significantly improved OS (10.1 months vs. 7.4 months, $\mathrm{P}=0.04)$ and progression free survival (PFS, 5.6 months vs. 3.3 months, $\mathrm{P}<0.001$ ) compared to the chemotherapy alone arm. While this was the first study to demonstrate significantly improved OS in this patient population, the EXTREME regimen is not without adverse effects. Patients in the cetuximab arm experienced 
significantly higher rates of sepsis (including septic shock) and skin reactions compared to patients receiving chemotherapy alone. Despite FDA approval and a Category 1 recommendation in the NCCN guidelines, use of the platinum, 5-FU, cetuximab regimen is limited in clinical practice due to toxicity. The KEYNOTE-048 trial introduced immunotherapy into the first-line setting for R/M SCCHN. This trial assessed 882 patients who received either pembrolizumab monotherapy, standard chemotherapy with the EXTREME regimen, or pembrolizumab plus a platinum and 5-FU. Pembrolizumab monotherapy and pembrolizumab plus chemotherapy led to a significant improvement in OS with a median of 11.5 and 13 months, respectively, compared to the EXTREME regimen at 10.7 months. Grade 3-5 adverse events were seen in $54.7 \%$ with pembrolizumab, $85.1 \%$ with pembrolizumab plus chemotherapy, and $83.3 \%$ with the EXTREME regimen.

An alternative to the EXTREME regimen for first-line treatment of $\mathrm{R} / \mathrm{M}$ SCCHN is a regimen containing a platinum, taxane, plus cetuximab. In the TPEx trial, 54 patients received four cycles of cisplatin, docetaxel, and cetuximab every 3 weeks followed by cetuximab maintenance therapy every two weeks thereafter (6). Overall response rates were $44.4 \%$ with a median PFS of 6.2 months and a median OS of 14 months. Although these results were promising, in clinical practice carboplatin and paclitaxel are often substituted for cisplatin and docetaxel, respectively, due to enhanced tolerability (1). Narveson et al is the only study that evaluated the weekly administration schedule of carboplatin and paclitaxel (3 weeks on, 1 week off for a maximum of six cycles, with weekly cetuximab continued during and after completion of chemotherapy) in 41 patients with very advanced or metastatic head and neck cancer (7). Partial responses were achieved in $37 \%$ of patients, with a median PFS of 4.6 months and median OS of 5.25 months. Although PFS and OS were lower in this study compared to the TPEx trial, the authors commented that 96\% of patients had an Eastern Cooperative Oncology Group (ECOG) performance status of 1 or 2 , indicating an inferior baseline performance status compared to patients typically eligible for clinical trials.

Standard practice at our institution is to administer paclitaxel, carboplatin, and cetuximab (PCC) as the first/second-line regimen of chemotherapy choice in the $\mathrm{R} / \mathrm{M}$ setting for patients with SCCHN, with chemotherapy administered weekly or every 3 weeks depending on patient's age, performance status, comorbidities, and physician preference. The weekly regimen studied by Narveson et al (7) uses higher doses given 3 weeks on, 1 week off. The break was included to help limit toxicity. Our institution's protocol uses lower doses of carboplatin and paclitaxel given weekly with no break to limit toxicity while providing a convenient schedule as cetuximab is administered weekly. Therefore, the primary objective of this study was to evaluate safety and toxicity outcomes in patients receiving weekly vs. every 3 weeks PCC. Secondary objectives were to compare median weekly relative dose intensity, PFS, and OS between groups.

\section{Materials and methods}

Treatment protocols. This was a retrospective, single-center, cohort study evaluating patients who had received treatment with weekly or every 3 weeks paclitaxel, carboplatin, and cetuximab. This study included patients with a diagnosis of R/M SCCHN who were 18 years of age and older. Patients received at least 3 weeks (one cycle) of the weekly regimen of PCC (paclitaxel $45 \mathrm{mg} / \mathrm{m}^{2}$ IV weekly + carboplatin AUC $1.5 \mathrm{IV}$ weekly + cetuximab $400 \mathrm{mg} / \mathrm{m}^{2} \mathrm{IV}$ week 1 , then $250 \mathrm{mg} / \mathrm{m}^{2} \mathrm{IV}$ weekly thereafter) or one cycle of the every 3 weeks regimen of PCC (paclitaxel $175 \mathrm{mg} / \mathrm{m}^{2} \mathrm{IV}$ every 3 weeks + carboplatin AUC 5 IV every 3 weeks + cetuximab $400 \mathrm{mg} / \mathrm{m}^{2}$ IV week 1 , then $250 \mathrm{mg} / \mathrm{m}^{2}$ IV weekly thereafter).

Study population. Patients who were prisoners, pregnant, diagnosed with nasopharyngeal carcinoma or salivary gland carcinoma, changed from paclitaxel to docetaxel, or received PCC as an induction regimen prior to radiation or surgery were excluded. All eligible patients treated at The James Cancer Hospital at The Ohio State University from January 1, 2013 to July 31, 2018 were included. Patients were stratified by age ( $\geq 65$ or $<65$ years); performance status (ECOG $0-1$ vs. ECOG 2-3); line of therapy (1st vs. 2nd and beyond in the R/M SCCHN setting); and those who recurred/progressed within 6 months of primary chemoradiation.

This study was approved by The James Cancer Hospital at The Ohio State University's Investigational Review Board and Clinical Scientific Review Committee. Data were collected for patients who met the pre-specified inclusion criteria. Data extracted from electronic medical records included patient demographics, disease characteristics, chemotherapy dosing, and toxicity considerations.

Toxicity evaluation. Toxicity was graded utilizing the Common Terminology Criteria for Adverse Events (CTCAE) version 4.03 and was determined as documented by the treating oncologist and review of the electronic medical record (8). PFS was defined as time from first dose of PCC to progression (as determined by oncologist's documentation in the electronic medical record, radiologist interpretation of imaging, and/or change in therapy) or death from any cause. OS was defined as time from first dose of PCC to death from any cause. Analysis time was censored at the last follow-up date for patients without PFS/OS events. Relative dose intensity (RDI) was defined as the cumulative dose of the agent delivered during treatment in milligrams per square meter $\left(\mathrm{mg} / \mathrm{m}^{2}\right)$ divided by the intended dose in $\mathrm{mg} / \mathrm{m}^{2}$ multiplied by 100 . This was reported as a percentage and determined for each agent: paclitaxel, carboplatin, cetuximab.

Grade 3/4 toxicity rates, PFS, and OS were compared between the weekly and every 3 weeks PCC treatment groups using inverse probability of treatment weighting (IPTW) to account for potential differences in patient characteristics between the groups. IPTW is a propensity score-based methodology that yields an unbiased treatment effect estimate. In this analysis, propensity scores for IPTW were calculated using probabilities from a logistic regression model with treatment regimen as the outcome and age, sex, race, baseline ECOG performance status, tobacco use, previous radiation, previous surgery, PCC initiation within 6 months of chemoradiation, and line of therapy in $\mathrm{R} / \mathrm{M}$ setting as the independent variables. Standardized differences between groups for patient characteristics were evaluated with and without application of 
IPTW to assess the balance achieved by IPTW. Although not absolute, one rule of thumb is that a standardized difference of $>0.2$ suggests imbalance between groups.

An IPTW-adjusted logistic regression model with IPTW was used to test group differences in incidence of grade 3/4 toxicities. The treatment group comparison of Grade 3/4 toxicity rates in the logistic regression model was assessed at the $\alpha=0.05$ significance level. Kaplan-Meier curves with IPTW were generated for each group for PFS and OS outcomes. Since the PFS and OS outcomes were hypothesized not to differ between the treatment regimens, statistical hypothesis tests were not performed for these outcomes. A non-significant result for these outcomes would not lead to the conclusion of equivalence or non-inferiority, and the sample size was inadequate for sufficiently powered statistical tests of equivalence or non-inferiority. Therefore, the PFS and OS outcomes were presented descriptively by the Kaplan-Meier curves and median time to event and/or event estimates at select follow-up times for each treatment group. Chemotherapy dose intensity, frequency of discontinuation, dose reductions, and dose delays were reported for each group.

In order to characterize PFS and OS for high-risk subgroups receiving the weekly $\mathrm{PCC}$ regimen, median survival and estimated survival at 1 year were calculated for patients receiving the weekly PCC treatment group with age $>65$, baseline ECOG of 2 or 3 , line of therapy $>1$, and recurrence/progression within 6 months of primary chemoradiation. Since there was no direct comparison group for these subgroups, the survival estimates were unweighted.

\section{Results}

Patient characteristics. A total of 207 patients with R/M SCCHN were screened for inclusion (Fig. 1). 133 patients were excluded with the most common reasons being that the patients did not receive all 3 PCC agents with the regimen, PCC was used as an induction regimen prior to surgery or radiation, the patients did not receive one complete cycle of PCC, or patients had nasopharyngeal or salivary sites of disease. As a result, 74 patients were included with 26 patients in the weekly PCC cohort and 48 patients in the every 3 weeks PCC cohort.

Table I illustrates baseline characteristics. The majority of the patients were males, white, and had received prior chemotherapy. The oropharynx was the most common site of disease for patients in both the weekly PCC group at 54\% and the every 3 weeks PCC group at $56 \%$. Of the 14 patients with disease of the oropharynx in the weekly PCC group, 6 (43\%) were HPV positive and 9 (64\%) were p16 positive. Of the 27 patients with disease of the oropharynx in the every 3 weeks PCC group, $12(44 \%)$ were HPV positive and $14(52 \%)$ were p16 positive. Prior to IPTW adjustment, differences between groups (Std. Diff $>0.2$ ) at baseline were notable as follows: the weekly PCC group compared to the every 3 weeks PCC group included more patients who were older (median 65 years of age vs. 60), white (92 vs. 85\%), had a worse ECOG performance status of 2 or 3 (27 vs. 17\%), were more heavily pretreated with radiation (92 vs. $85 \%$ ) and surgery (58 vs. $40 \%$ ), had PCC initiated within 6 months of chemoradiation (46 vs. 27\%), and received PCC as third line or greater for R/M disease (23 vs. $2 \%$ ). However, after IPTW adjustment, study groups were similar with the exception

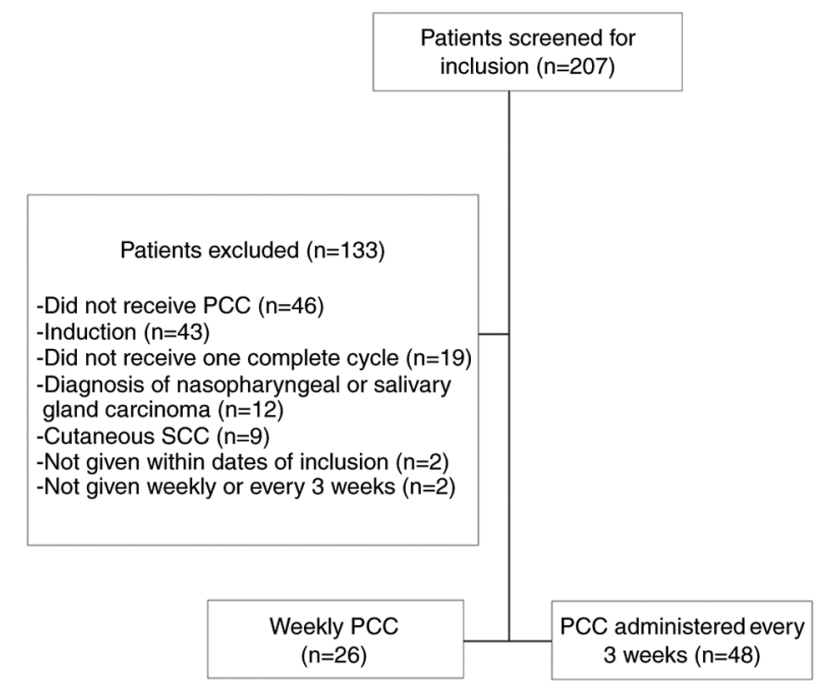

Figure 1. Patient collection. A total of 207 patients were screened for inclusion. Of these, 133 patients were excluded with the most common reason being that the patients did not receive all 3 PCC agents as part of their treatment regimen, or that $\mathrm{PCC}$ was used as an induction regimen prior to surgery or radiation. A total of 74 patients were included with 26 patients in the group administered PCC weekly and 48 patients in the group administered PCC every 3 weeks. PCC, paclitaxel, carboplatin and cetuximab; SCC, squamous cell carcinoma.

of the weekly PCC group having an older population (median 63 years of age vs. 60) and being more heavily pretreated with radiation (95 vs. $89 \%$ ) and surgery (58 vs. $46 \%$ ).

Toxicities. The incidence of grade $3 / 4$ toxicity was greater in the every 3 weeks PCC group at $66 \%$ compared to the weekly PCC group at $25 \%(\mathrm{P}=0.01)$ (Table II). The odds of having a grade $3 / 4$ toxicity was $82 \%$ lower with weekly PCC compared to every 3 weeks PCC [HR 0.18 (0.05-0.64)]. The most common grade $3 / 4$ adverse events seen in the every 3 weeks group vs. the weekly group were neutropenia (53 vs. $8 \%$ ), anemia (32 vs. $15 \%$ ), and fatigue (10 vs. $3 \%$ ). The incidence of grade $3 / 4$ toxicity or any grade toxicity requiring dose modification or discontinuation was $77 \%$ in the every 3 weeks PCC group vs. $74 \%$ in the weekly $\mathrm{PCC}$ group $(\mathrm{P}=0.78)$.

Survival. The median PFS in the weekly PCC group was 3.9 months compared to 5.3 months in the every 3 weeks PCC group. The 12-month PFS rate was 27 vs. 13\%, respectively (Fig. 2A). The median OS in the weekly PCC group was 7.6 months compared to 11.1 months in the every 3 weeks PCC group. The 12-month OS rate was $46 \%$ compared to $44 \%$ (Fig. 2B). The overall response rate was 39\% in the weekly PCC group vs. $27 \%$ in the every 3 weeks PCC group. No formal statistical comparisons were performed on secondary outcomes due to small sample size for a non-inferiority hypothesis.

Subgroup analyses assessing survival outcomes of patients in the weekly PCC group included patients who were $>65$ years old, had a poor performance status of 2 or 3 , or received PCC as a second line therapy or greater in the R/M setting (Table III). The median PFS for these subgroups was 4.8 months for patients $>65$ years old, 6.1 months for patients with a poor performance status of 2 or $3,3.9$ months for patients who 
Table I. Baseline characteristics.

\begin{tabular}{|c|c|c|c|c|c|c|}
\hline \multirow[b]{2}{*}{ Characteristic } & \multicolumn{3}{|c|}{ Unweighted } & \multicolumn{3}{|c|}{ IPTW Adjustment } \\
\hline & $\begin{array}{l}\text { Weekly } \\
(n=26)\end{array}$ & $\begin{array}{l}\text { Every } 3 \text { weeks } \\
\qquad(\mathrm{n}=48)\end{array}$ & $\begin{array}{l}\text { Std. } \\
\text { diff }^{\mathrm{a}}\end{array}$ & $\begin{array}{l}\text { Weekly } \\
(\mathrm{n}=23.7)\end{array}$ & $\begin{array}{l}\text { Every } 3 \text { weeks } \\
\qquad(n=45.6)\end{array}$ & $\begin{array}{l}\text { Std. } \\
\text { diff }^{\mathrm{a}}\end{array}$ \\
\hline Age, median (IQR) & $64.5(60-71)$ & $59.5(54-64)$ & 0.89 & $63(59-65)$ & $60(53-64)$ & 0.56 \\
\hline Sex, male & $22(84.6)$ & $40(83.3)$ & 0.04 & $22(91.1)$ & $39(86.4)$ & 0.15 \\
\hline Race & & & 0.22 & & & 0.03 \\
\hline Black & $2(7.7)$ & $6(12.5)$ & & $3(14.2)$ & $5(10)$ & \\
\hline White & $24(92.3)$ & $41(85.4)$ & & $20(85.8)$ & $40(86.8)$ & \\
\hline Baseline ECOG PS & & & 0.25 & & & 0.09 \\
\hline $0-1$ & $19(73.1)$ & $40(83.3)$ & & $18(77.2)$ & $37(80.7)$ & \\
\hline $2-3$ & $7(26.9)$ & $8(16.7)$ & & $5(22.8)$ & $9(19.3)$ & \\
\hline Primary site of disease & & & 0.33 & & & 0.26 \\
\hline Oral cavity & $6(23.1)$ & $7(14.6)$ & & $5(20.9)$ & $7(14.5)$ & \\
\hline Oropharynx & $14(53.8)$ & $27(56.3)$ & & $13(56.6)$ & $26(56.8)$ & \\
\hline Pharynx & $1(3.8)$ & $3(6.3)$ & & $0(1.5)$ & $2(5.4)$ & \\
\hline Larynx & $2(7.7)$ & $7(14.6)$ & & $3(14.5)$ & $7(16.4)$ & \\
\hline Paranasal & $3(11.5)$ & $4(8.3)$ & & $2(6.6)$ & $3(7)$ & \\
\hline Tobacco use & $19(73.1)$ & $31(64.6)$ & 0.18 & $18(77.1)$ & $31(68.5)$ & 0.19 \\
\hline Alcohol use & $9(34.6)$ & $19(39.6)$ & 0.10 & $10(41.6)$ & $19(41.8)$ & 0.01 \\
\hline \multicolumn{7}{|l|}{ Previous treatment } \\
\hline Chemotherapy & $20(76.9)$ & $37(77.1)$ & 0.01 & $19(79.4)$ & $37(82.3)$ & 0.07 \\
\hline Radiation & $24(92.3)$ & $41(85.4)$ & 0.22 & $23(95.3)$ & $40(88.9)$ & 0.24 \\
\hline Surgery & $15(57.7)$ & $19(39.6)$ & 0.37 & $14(57.6)$ & $21(45.6)$ & 0.24 \\
\hline $\begin{array}{l}\text { PCC initiated within } 6 \text { months } \\
\text { of chemoradiation }\end{array}$ & $12(46.2)$ & $13(27.1)$ & 0.4 & $9(36.1)$ & $14(31.7)$ & 0.09 \\
\hline $\begin{array}{l}\text { Line of therapy in a recurrent/metastatic } \\
\text { setting }\end{array}$ & & & 0.71 & & & 0.15 \\
\hline First & $18(69.2)$ & $45(93.8)$ & & $20(84.9)$ & $41(88.9)$ & \\
\hline Second & $2(7.7)$ & $2(4.2)$ & & $1(4.6)$ & $2(4.7)$ & \\
\hline Third or greater & $6(23.1)$ & $1(2.1)$ & & $3(10.6)$ & $3(6.3)$ & \\
\hline
\end{tabular}

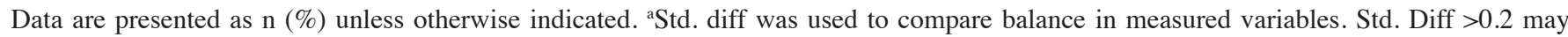
indicate imbalance. ECOG PS, Eastern Cooperative Oncology Group Performance Status; IGR, indeterminate range; IPTW, inverse probability of treatment weighting; PCC, Paclitaxel, Carboplatin and Cetuximab; Std. diff, standardized difference.

received PCC as a second line therapy or greater in the $\mathrm{R} / \mathrm{M}$ setting, and 6.1 months for patients who recurred/progressed within 6 months of primary chemoradiation compared to the median PFS for the weekly PCC cohort overall at 4.8 months. The PFS rates at 1 year were $0,14,0$, and $17 \%$ respectively, which were similar to the weekly PCC cohort overall at $18 \%$. The median OS was similar for each subgroup at 6.3, 7.6, 6.9, and 7.6 months compared to the weekly PCC cohort overall at 7.3 months. However, the OS rates at 1 year were $21,14,38$, and $31 \%$ respectively, similar to the $32 \%$ seen in the weekly PCC cohort overall.

The median RDI of paclitaxel for the weekly PCC group was 61 vs. $100 \%$ in the every 3 weeks PCC group. The RDI of carboplatin was 68 vs. $100 \%$ and the RDI of cetuximab was 83 vs. $94 \%$, respectively (Fig. 3). A total of 31 patients in the every 3 weeks PCC group were able to receive $100 \%$ of the doses intended leading to the high median in this group. Of the 17 patients with documented progression in the weekly PCC group, 12 received immunotherapy as a next line of treatment at progression. Zero patients were candidates for further chemotherapy at progression and 5 patients chose not to continue with a next line of therapy. Of the 35 patients with documented progression in the every 3 weeks PCC group, 8 received immunotherapy as a next line of treatment at progression. A total of 14 patients were candidates for further chemotherapy at progression and 13 patients chose not to continue with a next line of therapy.

\section{Discussion}

This retrospective study of the treatment of R/M SCCHN showed that a weekly PCC regimen was associated with lower toxicity compared to an every 3 weeks regimen of PCC. The odds of having a grade $3 / 4$ toxicity was $82 \%$ higher with every 
Table II. Toxicity with inverse probability of treatment weighting adjustment.

\begin{tabular}{lcc}
\hline Outcome & $\begin{array}{c}\text { Weekly } \\
(\mathrm{n}=23.7)\end{array}$ & $\begin{array}{c}\text { Every 3 weeks } \\
(\mathrm{n}=45.6)\end{array}$ \\
\hline $\begin{array}{l}\text { Grade 3/4 toxicity, }(\%)^{\mathrm{a}} \\
\text { Specific Grade 3/4 toxicity, n (\%) }\end{array}$ & $6(25.4)$ & $30(65.7)$ \\
Neutropenia & $2(7.6)$ & $24(52.5)$ \\
Thrombocytopenia & $0(0.0)$ & $3(6.6)$ \\
Anemia & $4(15.1)$ & $14(31.7)$ \\
Fatigue & $1(2.7)$ & $5(10)$ \\
Skin rash & $<1(1.7)$ & $0(0)$ \\
Neuropathy & $0(0)$ & $1(1.9)$ \\
Other & $<1(1.7)$ & $1(1.5)$ \\
Grade 3/4 toxicity or toxicity & $18(73.8)$ & $35(77.0)$ \\
requiring dose modification or & & \\
discontinuation, $\mathrm{n}(\%)^{\mathrm{b}}$ & & \\
\hline
\end{tabular}

adds ratio $(95 \% \mathrm{CI}), 0.18(0.05-0.64)$ corresponding to $\mathrm{P}=0.01$. bdds ratio $(95 \% \mathrm{CI}), 0.84(0.24-2.95)$ corresponding to $\mathrm{P}=0.78$.
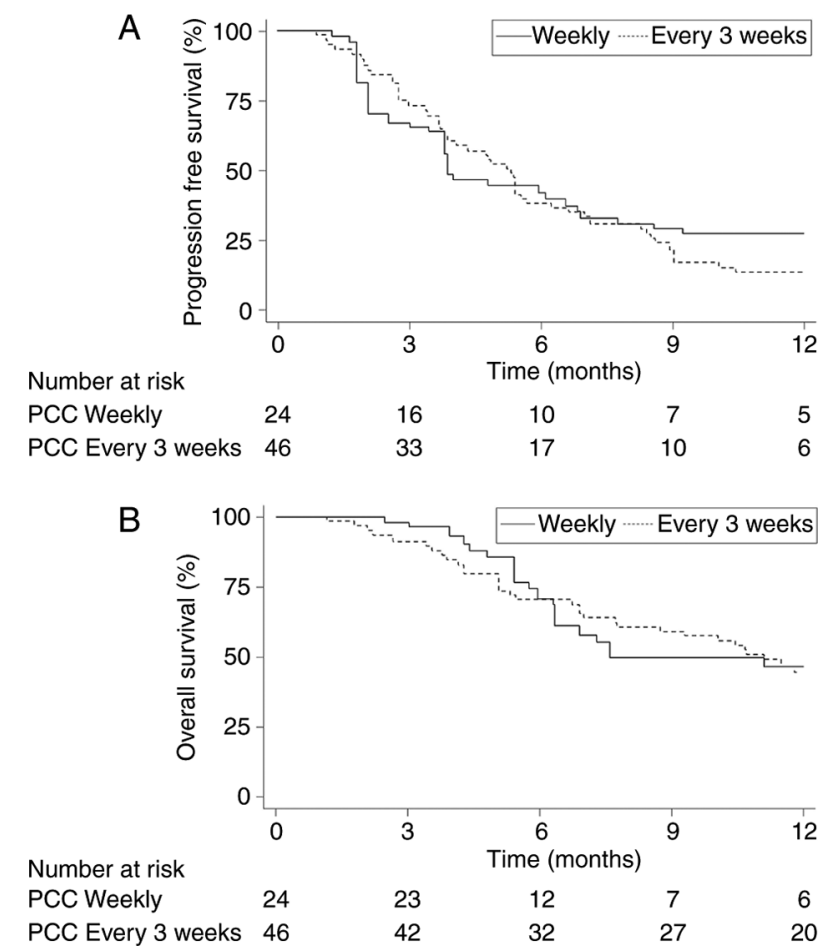

Figure 2. Survival curves for weekly and every 3-week PCC regimens. (A) PFS with IPTW adjustment. Median PFS was 3.9 months for the weekly PCC group compared to 5.3 months in the every 3 week PCC group. The one-year PFS rate was $27 \%$ in the weekly PCC group vs. $13 \%$ in the every 3 weeks PCC group. (B) OS with IPTW adjustment. Median OS was 7.6 months for the weekly PCC group compared to 11.1 months in the every 3 weeks PCC group. The one-year PFS rate was $46 \%$ in the weekly PCC group vs. $44 \%$ in the every 3 weeks PCC group. PFS, progression-free survival; IPTW, inverse probability of treatment weighting; PCC, paclitaxel, carboplatin and cetuximab; OS, overall survival

3 weeks PCC, and the most common adverse events seen in this group were neutropenia, anemia, and fatigue. This is consis- tent with previous trials for R/M SCCHN. In the TPEx trial, patients received paclitaxel $175 \mathrm{mg} / \mathrm{m}^{2}$ every 3 weeks in the PCC regimen with a $6 \%$ incidence of grade 4 neutropenia (6). At The James Cancer Hospital at The Ohio State University, the every 3 weeks PCC regimen had a higher incidence of grade $3 / 4$ neutropenia at $53 \%$. Narveson et al utilized a weekly PCC regimen with a dose of paclitaxel of $80 \mathrm{mg} / \mathrm{m}^{2}$ given 3 weeks on, 1 week off. The total cumulative dose for a cycle was $240 \mathrm{mg} / \mathrm{m}^{2}$, resulting in $39 \%$ of patients experiencing grade 3/4 neutropenia (7). Comparing the Narveson et al weekly regimen to the weekly regimen used in this study, PCC uses lower doses of paclitaxel $45 \mathrm{mg} / \mathrm{m}^{2}$ weekly. Since the total cumulative dose of paclitaxel is $135 \mathrm{mg} / \mathrm{m}^{2}$ each cycle, this could explain why the incidence of grade $3 / 4$ neutropenia was only $8 \%$ and lower than seen with any other group studied.

Secondary efficacy outcomes were assessed in each of these trials in the R/M setting. At The James Cancer Hospital, patients with a good performance status are considered to be able to tolerate the toxicities associated with the every 3 weeks PCC regimen, while patients with worse performance status and poor performance status are thought to derive more benefit from the weekly PCC regimen. As a result, the every 3 weeks PCC group had a median OS of 11.1 months, OS rate at 1 year of $44 \%$, median PFS of 5.3 months, PFS rate at 1 year of $13 \%$, and an ORR 27\%. This is comparable to the TPEx trial which resulted in a median OS of 15.3 months, median PFS of 7.1 months, and an ORR of 44\% (6). In contrast, the weekly PCC regimen was studied by Narveson et al in patients with poorer ECOG performance status of 1-2. This regimen resulted in a median OS of 5.25 months, median PFS of 4.6 months, and an ORR of $41 \%$ (7). The weekly PCC group for the purposes of this current study had a median OS of 7.6 months, OS rate at 1 year of $46 \%$, median PFS of 3.9 months, PFS rate at 1 year of $27 \%$, and an ORR $39 \%$. While the every 3 weeks PCC group had a numerically higher median OS than the weekly PCC group (11.1 mos vs. $7.6 \mathrm{mos}$ ), the OS rates at 1 year were similar (46 vs. 44\%), indicating that weekly PCC is a safe and effective option for patients with poor performance status and older age.

It was important to stratify the weekly cohort into high-risk subgroups as the practice at The James Cancer Hospital is to give the weekly PCC regimen to patients who were older, had a poor performance status, and/or were more heavily pretreated. The results of the subgroup analysis indicates that these patients achieved similar survival outcomes as the overall cohort receiving the weekly regimen. The weekly PCC regimen provides a treatment option for older patients with worse performance status who are heavily pretreated and would not be able to receive every 3 weeks PCC due to toxicity. There is very limited data on the use of weekly carboplatin and paclitaxel in combination with cetuximab, especially in the second line setting.

The patients in the every 3 weeks PCC group were able to maintain higher median dose intensity compared to the weekly PCC regimen throughout treatment and received more chemotherapy as a result. A reason for the difference in RDI relates to how the regimens are scheduled. The every 3 weeks PCC group was seen weekly due to cetuximab being given every week. Labs were drawn and systems assessments were completed that often showed grade 3/4 toxicity during 
Table III. Subgroup analysis of Paclitaxel, Carboplatin and Cetuximab treatment.

\begin{tabular}{lcccc}
\hline Unweighted & $\begin{array}{c}\text { Median PFS } \\
\text { (months) }\end{array}$ & $\begin{array}{c}\text { PFS rate at } \\
\text { 1 year (\%) }\end{array}$ & $\begin{array}{c}\text { Median OS } \\
\text { (months) }\end{array}$ & $\begin{array}{r}\text { OS rate at } \\
1 \text { year }(\%)\end{array}$ \\
\hline Age $>65(\mathrm{n}=13)$ & 4.8 & 0 & 6.3 & 20.5 \\
Baseline ECOG PS value of 2 or 3 $(\mathrm{n}=7)$ & 6.1 & 14.3 & 7.6 & 14.3 \\
Line of therapy $>$ 1 (n=8) & 3.9 & 0 & 6.9 & 37.5 \\
$\begin{array}{l}\text { Recurred/progressed within 6 months of primary } \\
\text { chemoradiation (n=12) }\end{array}$ & 6.1 & 16.7 & 7.6 & 31.3 \\
Full weekly cohort (n=26) & 4.8 & 18.5 & 7.3 & 32.1 \\
\hline
\end{tabular}

ECOG PS, Eastern Cooperative Oncology Group Performance Status; OS, overall survival; PFS, progression-free survival.

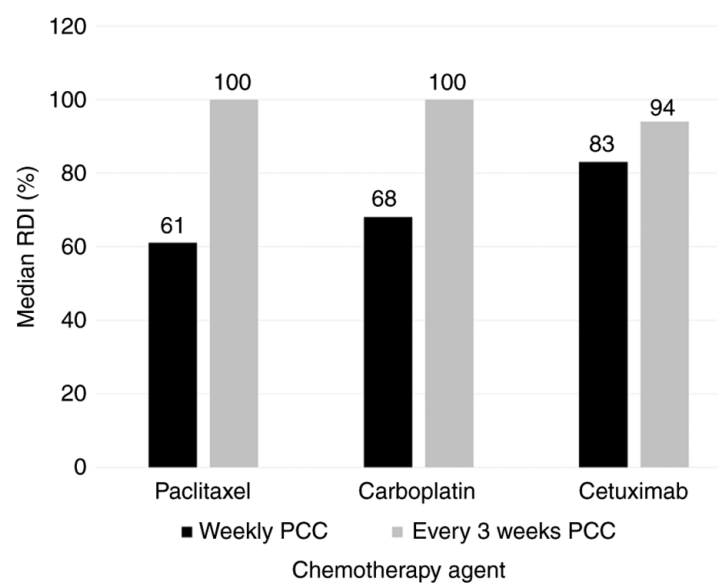

Figure 3. Median RDI. The median RDI of paclitaxel for the weekly PCC group was $61 \%$ compared with $100 \%$ in the every 3 weeks PCC group. Furthermore, the RDI of carboplatin and cetuximab was 68 and $83 \%$ in the weekly PCC group compared with 100 and $94 \%$ in the every 3 weeks PCC group, respectively. RDI, relative dose intensity; PCC, paclitaxel, carboplatin and cetuximab.

weeks 2 and 3 of a cycle. There were no dose modifications made as a result of toxicity as long as the toxicity resolved to grade $\leq 1$ by the start of the next cycle. However, the weekly regimen was seen each week to receive all 3 PCC agents. If there was toxicity seen on laboratory or systems assessment, dose modifications for grade 1/2 toxicity were more likely. As a result, this led to more toxicity in the every 3 weeks group but fewer dose reductions, leading to greater dose intensity. This rationale supports the reason why there was no difference between groups in toxicity when dose modifications were included because the weekly group was more likely to be dose reduced for grade $1 / 2$ toxicities.

There were several limitations to this study. It was a retrospective chart review, yielding a small sample size of 74 patients. Limited sample size is the major limitation of this study. Treatment selection was based not only on patients' performance status and age but also on physician preference which may have introduced bias. Incidences of adverse events as well as rationale for dose modifications were reliant on documentation in the medical record. However, one strength of this study was that the weekly regimen of PCC included a dosing strategy that has not previously been reported. Also, survival analysis on RDI was not performed as patients receiving weekly PCC were older, had worse performance status, were more heavily pretreated, and were more likely to experience a chemotherapy dose reduction if they experienced grade 2 toxicity.

In conclusion, the weekly $\mathrm{PCC}$ regimen represents a viable treatment option for R/M SCCHN due to an improved tolerability profile and similar efficacy compared to every 3 weeks PCC. In older, heavily pretreated patients with a decreased performance status, weekly PCC can help achieve goals of care by prolonging survival, improving quality of life, and limiting toxicity.

\section{Acknowledgements}

The abstract was presented at the American Society for Radiation Oncology (ASTRO) conference in Chicago, IL in September 2019 and published as abstract no. 351 in International Journal of Radiation Oncology on April 1, 2020.

\section{Funding}

No funding was received.

\section{Availability of data and materials}

All data generated or analyzed during this study are included in this published article.

\section{Author's contributions}

VK and MB conceived and designed the current study. LG prepared the original draft of the manuscript. LG, VK and MB wrote, reviewed and edited the manuscript. LG, MI, TES and SEH collected the data. LG and TES confirmed the authenticity of all the raw data. KP defined the statistical methodology, utilized statistical software tools and conducted formal statistical analysis. All authors read and approved the final version of the manuscript.

\section{Ethics approval and consent to participate}

The current research was conducted in accordance with the World Medical Association Declaration of Helsinki. As this 
is a retrospective study, the protocol was reviewed and the requirement for written and informed consent was waived by the Buck-Institutional Review Board of Ohio State University. The study protocol was approved by the aforementioned Institutional Review Board (Study ID, 2018C0169).

\section{Patient consent for publication}

Not applicable.

\section{Competing interests}

The authors declare that they have no competing interests.

\section{References}

1. Adelstein D, Gillison ML, Pfister DG, Spencer S, Adkins D, Brizel DM, Burtness B, Busse PM, Caudell JJ, Cmelak AJ, et al: NCCN guidelines insights: Head and neck cancers, version 2.2017. J Natl Compr Canc Netw 15: 761-770, 2017.

2. Chaturvedi AK, Engels EA, Anderson WF and Gillison ML: Incidence trends for human papillomavirus-related and -unrelated oral squamous cell carcinomas in the United States. J Clin Oncol 26: 612-619, 2008.

3. Duprez F, Berwouts D, De Neve W, Bonte K, Boterberg T, Deron P, Huvenne W, Rottey S and Mareel M: Distant metastases in head and neck cancer. Head Neck 39: 1733-1743, 2017.

4. Price KA and Cohen EE: Current treatment options for metastatic head and neck cancer. Curr Treat Options Oncol 13: 35-46, 2012.
5. Vermorken JB, Mesia R, Rivera F, Remenar E, Kawecki A Rottey S, Erfan J, Zabolotnyy D, Kienzer HR, Cupissol D, et al: Platinum-based chemotherapy plus cetuximab in head and neck cancer. N Engl J Med 359: 1116-1127, 2008.

6. Guigay J, Fayette J, Dillies AF, Sire C, Kerger JN, Tennevet I, Machiels JP, Zanetta S, Pointreau Y, Moal LB, et al: Cetuximab, docetaxel, and cisplatin as first-line treatment in patients with recurrent or metastatic head and neck squamous cell carcinoma: A multicenter, phase II GORTEC study. Ann Oncol 26: 1941-1947, 2015

7. Narveson L, Kathol E, Rockey M, Henry D, Grauer D and Neupane P: Evaluation of weekly paclitaxel, carboplatin, and cetuximab in head and neck cancer patients with incurable disease. Med Oncol 33: 107, 2016.

8. Common Terminology Criteria for Adverse Events (CTCAE). Version 4.03. US Department of Health and Human Services, National Institutes of Health and National Cancer Institute, 2010. https://evs.nci.nih.gov/ftp1/CTCAE/CTCAE 4.03/ CTCAE_4.03_2010-06-14_QuickReference_8.5x11.pdf. Accessed December 10, 2018.

(i) $(-)$ This work is licensed under a Creative Commons Attribution-NonCommercial-NoDerivatives 4.0 International (CC BY-NC-ND 4.0) License. 